\title{
In response to: Comparing strength of evidence: a rebuttal to A cluster- controlled trial of two prophylactic silicone sacral dressings to prevent sacral pressure injuries in critically ill patients
}

\author{
Stankiewicz M, Gordon J, Dulhunty JM, Brown W, Pollock H and Barker-Gregory N
}

For referencing Stankiewicz $\mathrm{M}$ et al. In response to: Comparing strength of evidence: a rebuttal to $A$ cluster-controlled trial of two prophylactic silicone sacral dressings to prevent sacral pressure injuries in critically ill patients. Wound Practice and Research 2020; 28(1):40-41.

DOI https://doi.org/10.33235/wpr.28.1.40-41

Dear Editor

In response to the critical review of our study by Brindle and Wright $^{1,2}$, we take the opportunity to provide several points of clarification. First, sample size calculations in our trial were based on a 'superiority vs. null hypothesis' two-sided

Monica Stankiewicz* RN, MAppSc (Research)

MNSc (NP), Grad Dip WM, Grad Cert STN

Nurse Practitioner

Royal Brisbane and Women's Hospital

Brisbane, QLD, Australia

Email mon.stankiewicz@gmail.com

Jodie Gordon RN, MClinSpec (WM)

MHealthAdmin, Grad Cert STN

Clinical Nurse Consultant

Wound Management, Stomal Therapy

Redcliffe Hospital, QLD

Joel M Dulhunty MBBS, MTH, PhD

Director of Research and Medical Education

Redcliffe Hospital, QLD

Wendy Brown RN, Cert ICN

Research Assistant, Intensive Care Unit

Redcliffe Hospital, QLD

Hamish Pollock BM, MRCP, FRCA, DICM(UK)

FCICM, FANZCA, PGDipCU

Director, Caboolture-Redcliffe Intensive Care Unit

Redcliffe Hospital, QLD

Nicola Barker-Gregory RN, Grad Cert ICU

Grad Cert Adult \& Wkplace Ed, M Edu

Project Officer, Redcliffe Hospital, QLD

* Corresponding author test, and not a non-inferiority approach as suggested by Brindle and Wright, which often employs use of a one-sided test $^{3}$. Given our use of a two-sided test for sample size calculations, this distinction is arguably irrelevant ${ }^{4}$. However, we agree with Brindle and Wright that our findings of no difference between the two dressings need to be interpreted with caution given the chance of a type 2 error associated with a smaller than estimated sample. We also agree that our findings may not be extrapolated to specific Intensive Care Unit (ICU) sub-populations not featured in this study that may be at higher risk of pressure injury development. We disagree with Brindle and Wright that our conclusions are not supported by our study design or findings taking into account the stated limitations ${ }^{2}$.

Brindle and Wright have queried the cluster design and its potential interaction with seasonal variables in our study. We acknowledge this remains a possibility, although there was no direct overlap with the recognised seasons in Australia $^{5}$. The three-month intervention cycles in our study were commenced on 17 February 2016 (Dressing 1), 11 May 2016 (Dressing 2), 17 August 2016 (Dressing 1), 16 November 2016 (Dressing 2), 15 February 2017 (Dressing 1) and 17 May 2017 (Dressing 2). All seasons were represented to some degree in both groups, although Dressing 1 (Allevyn Life ${ }^{\mathrm{TM}}$ ) was used more often in the Summer-Autumn period and Dressing 2 (Mepilex Border Sacrum ${ }^{\mathrm{TM}}$ ) in the Autumn-Winter period. While block allocation was chosen for logistical reasons in our ICU setting, we agree that individual patient randomisation allows for a higher degree of bias minimisation. Nevertheless, we found no evidence of difference between the two periods in terms of the baseline characteristics measured.

Brindle and Wright note a typographical error in our manuscript 
relating to the dressing used in one of the treatment arms (Dressing 1) that was corrected by an erratum ${ }^{6}$. The comparator dressing to Mepilex Border Sacrum ${ }^{\mathrm{TM}}$ (Dressing 2) was Allevyn Life ${ }^{\mathrm{TM}}$ as previously stated in the study protocol ${ }^{7}$, and not Allevyn Gentle Border Sacrum ${ }^{\mathrm{TM}}$ as initially reported in our paper ${ }^{2}$. The product description, dimensions and cost are, however, correctly reported.

We note the query by Brindle and Wright as to the difference in the total number of dressings per patient by study group (i.e. 1 and 2 dressings), despite similar patient daily dressing use (0.5 dressings) and dressing duration ( 2 days) in both treatment $\mathrm{arms}^{2}$. The reason for this apparent discrepancy is that median values are reported (in Table 2) for these variables due to their non-normal distribution.

Finally, we believe our paper is important because it provides comparative evidence from the first head-to-head comparison of two prophylactic sacral dressings in an ICU setting. Of note, our study has also been conducted independent of commercial sponsorship or affiliation. We thank Brindle and Wright for highlighting the significant healthcare cost savings that can be made by use of silicone foam dressings to prevent pressure injury development in comparison to standard care without dressings ${ }^{8}$, and agree that further comparative randomised controlled evidence of efficacy and cost effectiveness will contribute to clinician confidence in appropriate product selection.

\section{Conflict of interest}

The authors declare no conflicts of interest.

\section{Funding}

The authors received no funding for this study.

\section{References}

1. Brindle $T$, Wright E. Comparing strength of evidence: a rebuttal to "a cluster-controlled clinical trial of two prophylactic silicone sacral dressings to prevent sacral pressure injuries in critically ill patients". Wound Pract Res 2020; 28(1):38-39.

2. Stankiewicz M, Gordon J, Dulhunty J, Brown W, Pollock H, Barker-Gregory N. A cluster-controlled clinical trial of two prophylactic silicone sacral dressings to prevent sacral pressure injuries in critically ill patients. Wound Pract Res 2019;27(1):2126.

3. Scott IA. Non-inferiority trials: determining whether alternative treatments are good enough. Med J Aust 2009;190(6):326-330.

4. Dunn DT, Copas AJ, Brocklehurst P. Superiority and noninferiority: two sides of the same coin? Trials 2018;19(1):499.

5. Australian Government, Bureau of Meteorology. Seasons. Available from: http://www.bom.gov.au/climate/glossary/ seasons.shtml (accessed 20 February 2020).

6. Stankiewicz $\mathrm{M}$ et al. Errata for "A cluster-controlled clinical trial of two prophylactic silicone sacral dressings to prevent sacral pressure injuries in critically ill patients. WP\&R Journal 2019;27(1):21-26. Wound Pract Res 2019;27(2):61.
7. Gordon J, Stankiewicz M, Pollock H, Christensen M, BarkerGregory N, Dulhunty J. A trial of two prophylactic sacral dressings (2PSD) in the prevention of Stage 1 sacral pressure injury in the critically ill patient: A study protocol. Wound Pract Res 2017;25(2):82-86.

8. Santamaria N, Liu W, Gerdtz M et al. The cost-benefit of using soft silicone multilayered foam dressings to prevent sacral and heel pressure ulcers in trauma and critically ill patients: a withintrial analysis of the Border Trial. Int Wound J 2015;12(3):344-350. 